\title{
Sclerotinia sclerotiorum $\gamma$-Glutamyl Transpeptidase (Ss-Ggt1) Is Required for Regulating Glutathione Accumulation and Development of Sclerotia and Compound Appressoria
}

\author{
Moyi Li, ${ }^{1}$ Xiaofei Liang, ${ }^{2}$ and Jeffrey A. Rollins ${ }^{2}$ \\ ${ }^{1}$ Department of Molecular Genetics and Microbiology and ${ }^{2}$ Department of Plant Pathology, University of Florida, Gainesville \\ 32611, U.S.A.
}

Submitted 10 June 2011. Accepted 27 October 2011.

\begin{abstract}
Transcripts encoding Sclerotinia sclerotiorum $\gamma$-glutamyl transpeptidase (Ss-Ggt1) were found to accumulate specifically during sclerotium, apothecium, and compound appressorium development in $S$. sclerotiorum. To determine the requirement of this protein in these developmental processes, gene deletion mutants of $S$ s-ggt1 were generated and five independent homokaryotic $\Delta S s$-ggt1 mutants were characterized. All deletion mutants overproduced sclerotial initials that were arrested in further development or eventually produced sclerotia with aberrant rind layers. During incubation for carpogenic germination, these sclerotia decayed and failed to produce apothecia. Total glutathione accumulation was approximately 10-fold higher and $\mathrm{H}_{2} \mathrm{O}_{2}$ hyperaccumulated in $\Delta S s$-ggt1 sclerotia compared with the wild type. Production of compound appressoria was also negatively affected. On host plants, these mutants exhibited a defect in infection efficiency and a delay in initial symptom development unless the host tissue was wounded prior to inoculation. These results suggest that Ss-Ggt1 is the primary enzyme involved in glutathione recycling during these key developmental stages of the $S$. sclerotiorum life cycle but Ss-Ggt1 is not required for host colonization and symptom development. The accumulation of oxidized glutathione is hypothesized to negatively impact these developmental processes by disrupting the dynamic redox environment associated with multicellular development.
\end{abstract}

The Leotiomycetes fungus Sclerotinia sclerotiorum (Lib.) de Bary is a necrotrophic, broad-host-range plant pathogen of global agroeconomic importance (Boland and Hall 1994; Bolton et al. 2006; Purdy 1979). To initiate infection, S. sclerotiorum directly penetrates the healthy plant cuticle using a series of modified hyphae ranging from single-celled, unmelanized simple appressoria to multicellular compound appressoria, variably termed complex appressoria, secondary appressoria, lobate appressoria, multihyphal appressoria, infection cushions, and appressorial masses (Akutsu et al. 1983; Emmett and Parbery 1975; Purdy 1958; Tariq and Jeffries 1984). These compound appressoria arise from saprotrophic hyphae, adhere to the host

Corresponding author: J. A. Rollins; E-mail: rollinsj@ufl.edu; Telephone: +1.352 .273 .4620 ; Fax: +1.352.392.6532.

* The $\boldsymbol{e}$-Xtra logo stands for "electronic extra" and indicates that Figures 3,5 , and 6 appear in color online. surface, and grow through the healthy plant cuticle (Lumsden and Dow 1973). The microscopic features and developmental course of compound appressoria development in Sclerotiniaceae species has been documented on a number of hosts and artificial surfaces (Abawi et al. 1975; Akutsu et al. 1983; Backhouse and Willetts 1987; Cruickshank and Wadea 1992; Purdy 1958; Tariq and Jeffries 1984). Studies have further revealed involvement of nutrients (Cruickshank and Wadea 1992), surface hardness (Tariq and Jeffries 1984), and, more recently, cAMP signaling (Jurick and Rollins 2007) as regulators of compound appressorium development and complexity. Systematic studies to identify endogenous genetic regulators of compound appressoria development have not been reported.

Following successful cuticle penetration, subcuticular vesicles form and give rise to inter- and intracellular infectious hyphae which cause water soaking, necrosis, and complete collapse of host cells. Following host colonization, sclerotia form on the surface of or within the dead plant tissue (Abawi et al. 1975; Lumsden and Dow 1973). The sclerotium is formed from aggregates of pseudoparenchymatous hyphae with a continuous, generally single-cell layer of external, melanized cells. As longterm survival structures, sclerotia maintain viability of the fungus through environmental temperature extremes, low moisture, and high UV, and provide protection from microbial degradation and insect predation. Sclerotia formation is a crucial stage in the life cycle of $S$. sclerotiorum; therefore, numerous studies have identified molecules and signal transduction pathways involved in sclerotial initiation and development (Chet and Henis 1975; Erental et al. 2007). Among these, oxidative stress is reported as a common factor linked to sclerotial biogenesis in many sclerotium-forming fungi (Georgiou et al. 2006). In particular, accumulation of reactive oxygen species (ROS) and consequent lipid peroxidation are known to increase during sclerotial differentiation (Georgiou 1997; Papapostolou and Georgiou 2010). Consistent with a requirement for ROS accumulation to initiate and promote sclerotial development, scavengers of ROS inhibit sclerotial formation in S. sclerotiorum, S. minor, Sclerotium rolfsii, and Rhizoctonia solani (Georgiou 1997; Georgiou et al. 2006; Patsoukis and Georgiou 2008). The effect of oxidative stress on sclerotial initiation and involvement of thio-redox dynamics reported by Patsoukis and Georgiou $(2007,2008)$ further indicate significant involvement of thiols, including glutathione (GSH), in sclerotial development.

Reduced GSH is a major and ubiquitous antioxidant functioning to combat oxidative stress in animal and plant cells 
(May et al. 1998; Meister and Anderson 1983). In the presence of ROS, GSH can be oxidized to glutathione disulfide (GSSG) in oxidation-reduction reactions catalyzed by glutathione peroxidase. GSSG can, in turn, be reduced to reform GSH by glutathione reductase (Blokhina et al. 2003). GSH, GSSG, and GSSG/GSH ratios are widely reported to influence cell proliferation and differentiation in animal models (Franco and Cidlowski 2009; Krance et al. 2010; May et al. 1998; Pallardó et al. 2009). In filamentous fungi, a transient spike in oxidation known as the hyperoxidant state is associated with and hypothesized to play a functional role in the promotion of multicellular developmental transitions (Aguirre et al. 2005; Hansberg and Aguirre 1990; Scott and Eaton 2008).

Among the factors regulating GSH accumulation, $\gamma$-glutamyl transpeptidase (Ggt) (EC 2.3.2.2) catalyzes the transfer of a $\gamma$-glutamyl moiety of GSH, GSSG, and other c-glutamyl compounds to amino acids, small peptides, or water (Meister and Anderson 1983; Tate and Meister 1981). The transfer of a $\gamma$ glutamyl moiety is an essential first step in GSH metabolism and recycling and an important factor in maintaining redox homeostasis in the cellular environment (Zhang and Forman 2009). Characteristics of Ggt function and regulation in filamentous fungi have not been established but the encoded

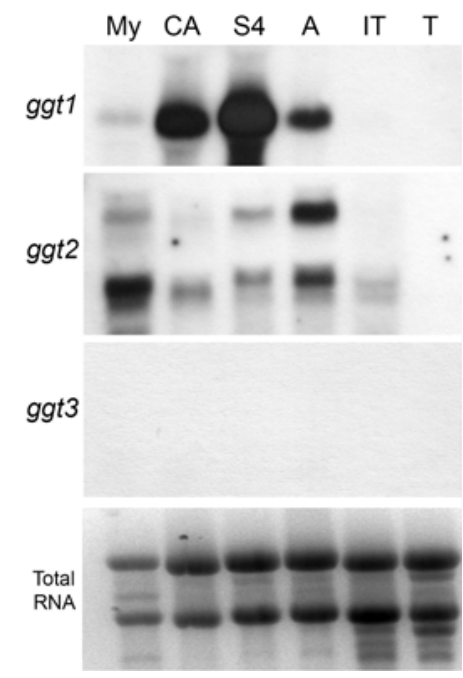

Fig. 1. Northern hybridization analysis of Sclerotinia sclerotiorum $\gamma$-glutamyl transpeptidase $S s$ - $g g t 1, S s$ - $g g t 2$, and $S s$-ggt3 transcript accumulation in different stages of wild-type development (My, mycelial suspension culture; CA, compound appressoria; $\mathrm{S} 4$, stage 4 consolidating sclerotia; A, developing apothecia; IT, infected tomato leaves; $\mathrm{T}$, healthy tomato leaves). enzymes are common to fungal genomes, including three predicted Ggt-encoding genes in S. sclerotiorum (Ss-ggt1, SS1G_ 14127.3; Ss-ggt2, SS1G_10940.3; and Ss-ggt3, SS1G_05330.3). Among these, Ss-ggtl was determined based on expressed sequence tag (EST) frequency (Amselem et al. 2011) to be transcriptionally upregulated during sclerotial initiation and compound appressorium development. The evidence for the regulation of the redox environment via thiols in sclerotia development (Patsoukis and Georgiou 2007, 2008) lead us to hypothesize that GSH recycling plays an important functional role in the development of sclerotia and other multicellular developmental stages. To test this hypothesis, we created gene deletion Ss-ggt1 mutants and determined the effects of Ss-Ggt loss of function on sclerotial and compound appressorium development and the host-pathogen interaction.

\section{RESULTS}

\section{Expression of Ss-ggt paralogs.}

Queries of the S. sclerotiorum genome sequence revealed three genes predicted to encode Ggt activity: SS1G_14127.3 (Ss-ggt1), SS1G_10940.3 (Ss-ggt2), and SS1G_05330.3 (Ssggt3). Homologs of all three Ss-ggt genes are conserved among plant, animal, and bacterial species. Analysis of the predicted protein sequences curated at the Fungal Secretome KnowledgeBase (Lum and Min 2011) predicts secretion signals and extracellular, non-membrane localization for Ss-Ggt1 and Ss-Ggt2 and cytoplasmic localization for Ss-Ggt3. SsGgt1 and Ss-Ggt2 share $47 \%$ amino acid identity and $62 \%$ similarity. The identity and similarity between Ss-Ggt3 and both Ss-Ggt1 and Ss-Ggt2 is approximately 24 and $40 \%$, respectively. RNAs extracted from several stages of the $S$. sclerotiorum development were used for Northern hybridization with coding sequence probes from each Ss-ggt gene to assess expression during different stages of the lifecycle. This analysis indicated that $S s$-ggtl transcript accumulation is very high during sclerotium, compound appressorium, and apothecium development but only weakly expressed or undetectable during vegetative mycelial growth and plant colonization (Fig. 1). Peak expression of Ss-ggtl was observed during stage 4 (the consolidation stage) of sclerotial development (Fig. 1 and data not shown). Analysis of Ss-ggt2 transcripts revealed the accumulation of two distinct size isoforms (Fig. 1). A high accumulation level of the small isoform was observed in vegetative mycelia and high levels of both isoforms was observed during apothecial development. Low levels of both isoforms were observed in developing sclerotia and low levels of only the small isoform were observed in compound appressoria and colonized tomato leaves. Very low, constitutive accumulation of $S s$-ggt3
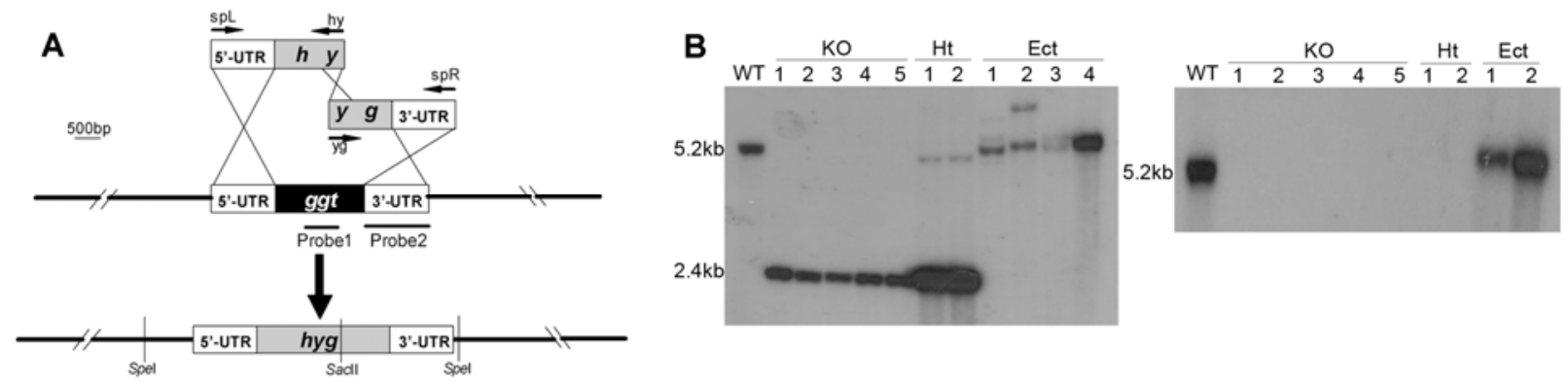

Fig. 2. A, "Split-marker" strategy used for creating Sclerotinia sclerotiorum $\gamma$-glutamyl transpeptidase (Ss-ggt1) deletion mutants and B, Southern hybridization for screening homokaryotic Ss-ggt1 gene knockout mutants (KO). Left panel, probe 2 hybridization of total genomic DNA double digested with SpeI and $\mathrm{Sac}$ I. The wild type (WT) hybridizes with a 5.2-kb band, transformants with a homologous integration at the Ss-ggtl locus hybridize with a 2.4-kb gene replacement band (KO), both bands hybridize with heterokaryotic (Ht) transformants, and bands of $5.2 \mathrm{~kb}$ and random sizes hybridize in ectopic (Ect) transformants. Right panel, probe 1 (coding sequence) hybridization of total genomic DNA double digested with SpeI and SacI. Note no hybridization Ss-ggt1 coding sequences with homokaryotic KO transformants and weak hybridization with the Ht transformants. 
transcript was observed in all of the fungal RNA samples (Fig. 1). No cross hybridization with uninoculated tomato leaves was observed with any of the three gene probes. The specific and high level of Ss-ggtl transcript accumulation during sclerotium, compound appressorium, and apothecium development suggested a role for Ss-Ggt in these important multicellular differentiation processes. On this basis, Ss-ggtl was chosen for further functional characterization. Ss-ggtl gene knockout strains were generated and effects of gene deletion on sclero-
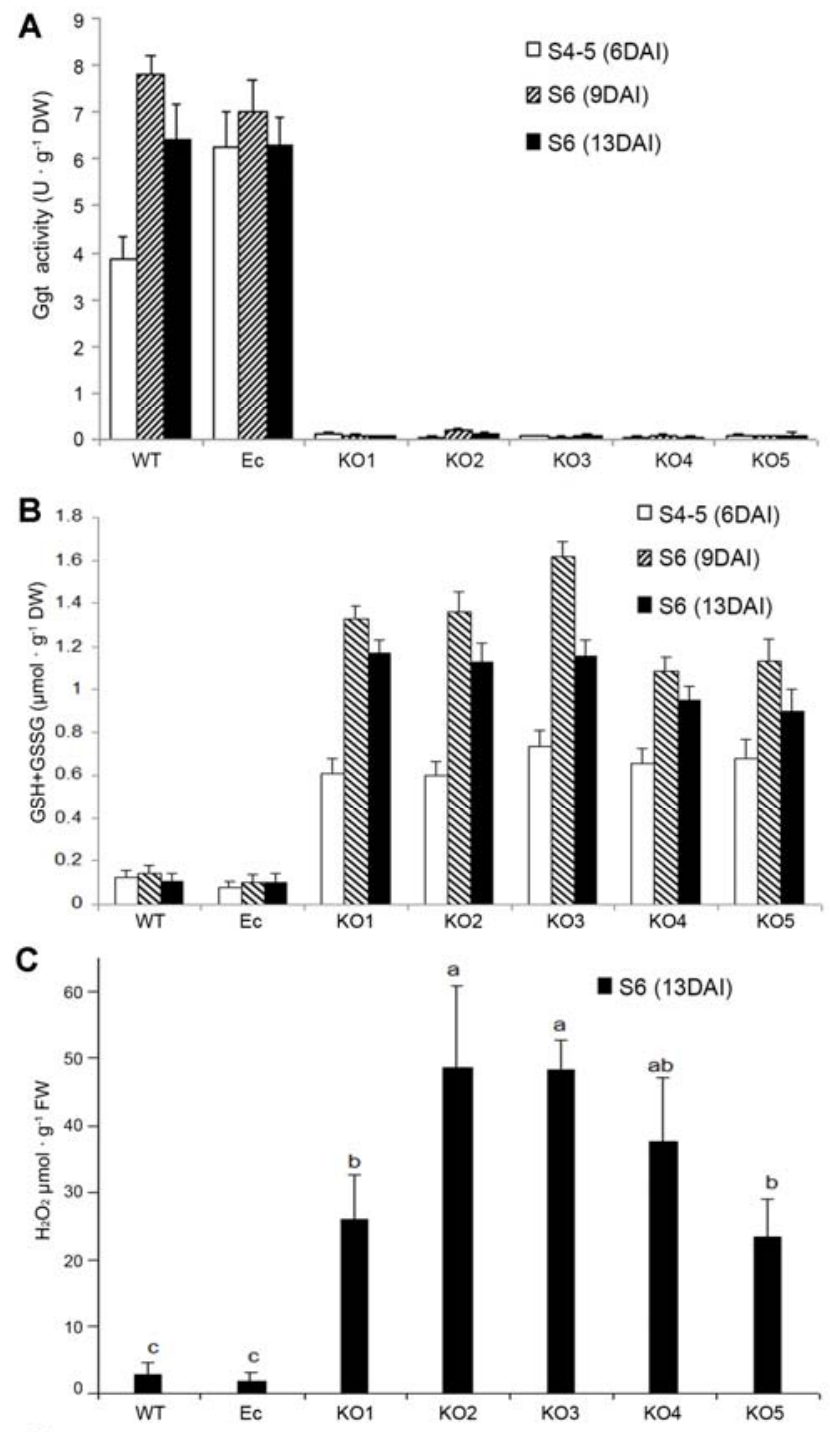

D

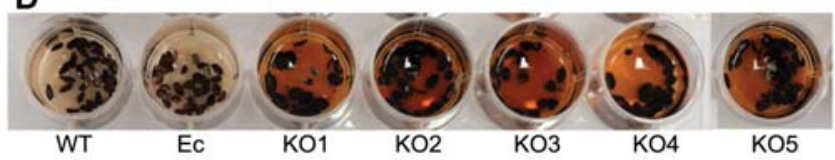

Fig. 3. Measurements of $\gamma$-glutamyl transpeptidase (Ggt) activity, glutathione (GSH) plus glutathione disulfide (GSSG), and hydrogen peroxide from sclerotia of wild type (WT), ectopic (Ec), and $\Delta S$ s-ggt1 knockout strains (KO1 to KO5). A, Ggt enzymatic activity was directly measured from sclerotial extracts. B, Total GSH (GSH+GSSG) accumulation in stage 4 to 6 (consolidating to mature) sclerotia. C, Hydrogen peroxide accumulation in stage 6 mature sclerotia. A to $\mathbf{C}$, Means and standard deviation values (1 SD) of three independent biological replications are shown. In B, the WT and ectopic values are significantly different from the five $S s$-ggt1 $\mathrm{KO}$ mutants at all three time points. In $\mathrm{C}$, bars not connected with the same letter are significantly different. Statistical inference was determined by Tukey's range test $(\alpha=0.05)$. D, 3,3'Diaminobenzidine staining of fresh, sliced stage 6 sclerotia. tial development and germination as well as development of compound appressoria and pathogenicity were investigated.

\section{Deletion of the Ss-ggt1 gene.}

The strategy employed for Ss-ggtl deletion is described below and is illustrated in Figure 2. The $3^{\prime}$-untranslated region (UTR) sequence and partial Ss-ggtl coding sequence were used as probes in Southern hybridization to screen for homokaryotic $S s-g g t 1$ knockout mutants. In all, 5 of 22 hygromycinresistant transformants were identified as homokaryotic $\mathrm{Ss}$ ggt1-deletion $\Delta S$ s-ggtl mutants (Fig. 2). Northern hybridization also indicated that there is no accumulation of Ss-ggtl transcripts in $\Delta S s$-ggtl sclerotia (data not shown). We characterized the phenotypes of all five of these independent gene deletion mutants relative to the wild type (WT) and an ectopic strain (ectopic).

\section{Physiological effects of $\mathrm{Ss}$-ggt1 deletion.}

To test whether deletion of $S$ s-ggtl reduces total Ggt activity and alters the accumulation of total GSH (GSH+GSSG) in developing sclerotia, stage 4 to 6 (consolidating to mature) sclerotia from the WT, ectopic, and $\Delta S$ s-ggtl mutants were assayed for Ggt activity and GSH+GSSG concentration. As hypothesized, all five independent $\Delta S s$-ggt1 mutants lost Ggt activity (Fig. 3A) and accumulated approximately 10-fold higher GSH+GSSG in stage 4 to 6 sclerotia relative to the WT and ectopic strain (Fig. 3B) (e.g., 9-day stage 6 sclerotia of the KO mutants averaged $1.3 \pm 0.2 \mu \mathrm{mol}$ GSH+GSSG per $g$ of dry weight versus an average of $0.1 \pm 0.01 \mu \mathrm{mol}$ GSH+GSSG per $\mathrm{g}$ of dry weight for the WT and ectopic). At all tested stages of sclerotial development, GSG+GSSG accumulation was significantly higher (Tukey's range test, $\alpha=0.05$ ) in the mutants relative to the WT and ectopic. Additionally, $\mathrm{H}_{2} \mathrm{O}_{2}$ concentration was both quantitatively (approximately 15-fold; WT $2.7 \pm$ $1.9 \mu \mathrm{mol} \mathrm{H}_{2} \mathrm{O}_{2}$ per $\mathrm{g}$ of dry weight; ectopic $2.2 \pm 1.3 \mu \mathrm{mol}$ $\mathrm{H}_{2} \mathrm{O}_{2}$ per $\mathrm{g}$ of dry weight; KO1-5 average $37 \pm 12 \mu$ mol $\mathrm{H}_{2} \mathrm{O}_{2}$ per $g$ of dry weight) and qualitatively higher in mature (stage 6) $\Delta S s$-ggt 1 sclerotia as assessed by the $3,3^{\prime}$ diaminobenzidine (DAB) treatment of sliced sclerotia (Fig. 3C and D).

\section{Effects of Ss-ggt1 deletion on development.}

The Ss-ggtl deletion affects both the timing of sclerotial initiation and the maturation of sclerotia but not the rate of hyphal growth. The WT and ectopic strain grown on potato dextrose agar (PDA) form stage 5 to 6 (pigmented and mature) sclerotia between 5 to 7 days after inoculation while $\Delta S s$-ggt 1 sclerotia remain in stage 4 to 5 (consolidating and pigmented) at this time (Fig. 4A). Moreover, from qualitative observations, $\Delta S s$-ggtl mutants produce greater numbers of sclerotial initials and condensing sclerotia (stages 1 to 2 ) than the WT and ectopic strain (Fig. 4A); however, most of these initials eventually senesce, such that the number of $\Delta S s$-ggtl sclerotia formed is not noticeably different from the WT or ectopic strain by 9 to 10 days.

In addition to the timing of sclerotial development, $S$ s-ggt 1 deletion also affects sclerotial tissue organization. Under the conditions of our assays, mature WT sclerotia generally contain a melanized rind composed of a single-cell layer. Mature sclerotia (stage 6) from $\Delta S s$-ggtl produced a thickened, multicellular, disorganized, melanized rind (Fig. 4B). Unlike the firm rind of WT sclerotia, the rind and cortex of fully mature $\Delta S s$-ggtl sclerotia could be easily peeled away from the medulla when harvested on or after 20 days from the date of plate inoculation (Fig. 4A). Microscopic examination of thin sections determined that the cortex layer of mature sclerotia of the mutants showed partial separation from the medulla. These mutant sclerotia were still able to myceliogenically germinate 
on agar media; however, the mature sclerotia of the Ss-ggt1 knockout mutants collected for carpogenic germination became soft and decayed during the incubation period and failed to germinate into apothecia after multiple independent attempts in which the WT and ectopic strain produced apothecia (results not shown).

\section{Effects of Ss-ggt1 deletion}

on compound appressoria and pathogenicity.

An in vitro assay was used to assess compound appressorium production in all strains. The WT and ectopic strain formed compound appressoria abundantly, as was shown macroscopically by aggregated hyphae and pigment accumulation associated with mature, compound appressoria (Fig. 5A). The $\Delta S s$-ggtl mutant strains failed to produce compound appressoria (Fig. 5A). To determine whether this developmental defect also occurred in planta, onion epidermal strips were inoculated with mycelial plugs and examined microscopically (Fig. 5B to E). The WT and the ectopic strain (not shown) produced compound appressoria, penetrated the onion epidermis, and produced infectious hyphae (Fig. 5B and D). The mutants initiated compound appressorium development, as shown by the production of hyphal tips that hook and bifurcate in a manner characteristic of compound appressorium initiation (Fig. 5C and E), but did not develop into tightly packed multihyphal compound appressoria. Microscopic examination of individual hyphae on cellophane revealed the same initiation but lack of compact, mature compound appressorium production in the mutant relative to WT (Fig. 5F versus G).

Inoculation of unwounded, detached tomato leaflets with $\Delta S s$-ggtl mutants revealed a decrease in infection efficiency as measured by the total number of infected leaflets, $47 \%(n=45)$, versus the WT and ectopic control, $100 \%(n=18)$, and delayed symptom development (Table 1; Fig. 6). Plants that were wounded prior to inoculation did not show a difference in infection efficiency: $98 \%(n=45)$ for $\Delta S$ s-ggtl mutants versus $100 \%(n=18)$ for WT and ectopic control. Likewise, symptom development with $\Delta S$ s-ggtl mutants was indistinguishable from the WT when leaflets were wounded prior to inoculation (Table 1; Fig. 6). The ectopic strain consistently appeared slightly more aggressive than other strains, including the WT, but overlapped statistically with the WT or $\Delta S s-g g t 1-\mathrm{KO} 2$ in lesion area on wounded leaflets (Table 1).

\section{DISCUSSION}

In this study, we have identified Ggt activity encoded by the Ss-ggtl gene as a developmentally regulated component of the $S$. sclerotiorum ROS antioxidant system. Sclerotia of loss-of-

Table 1. Mean lesion area in nonwounded and wounded tomato leaflets ${ }^{\mathrm{w}}$

\begin{tabular}{|c|c|c|c|c|}
\hline \multirow[b]{3}{*}{ Strain $^{y}$} & \multicolumn{4}{|c|}{ Area $\left(\mathrm{cm}^{2}\right)^{x}$} \\
\hline & \multicolumn{2}{|c|}{ Unwounded } & \multicolumn{2}{|c|}{ Wounded } \\
\hline & $24 \mathrm{~h}$ & $48 \mathrm{~h}$ & $24 h^{z}$ & $48 \mathrm{~h}$ \\
\hline WT & $44 \mathrm{a}, \mathrm{b}$ & $334 \mathrm{a}$ & 57 & $351 \mathrm{a}, \mathrm{b}$ \\
\hline $\mathrm{EC}$ & $55 \mathrm{a}$ & $431 \mathrm{a}$ & 93 & $525 \mathrm{a}$ \\
\hline KO1 & 8 b,c & $70 \mathrm{~b}$ & 54 & $346 a, b$ \\
\hline $\mathrm{KO} 2$ & $5 \mathrm{c}$ & $87 \mathrm{~b}$ & 70 & $447 a, b$ \\
\hline KO3 & $5 \mathrm{c}$ & $84 \mathrm{~b}$ & 44 & $281 \mathrm{~b}$ \\
\hline KO4 & $0 \mathrm{c}$ & $9 \mathrm{~b}$ & 59 & $402 \mathrm{a}, \mathrm{b}$ \\
\hline KO5 & $0 \mathrm{c}$ & $20 \mathrm{~b}$ & 48 & $340 a, b$ \\
\hline
\end{tabular}

${ }^{\mathrm{w}}$ Following an analysis of variance (ANOVA) with an overall $P=0.05$, the Bonferroni (Dunn) $t$ test with $\alpha=0.05$ was used to detect mean differences. Within columns, mean values with the same letter are not significantly different.

${ }^{x}$ Measured across three independent experiments with a total of nine reps per treatment.

y WT, wild type; EC, ectopic; KO1-KO5 Sclerotinia sclerotiorum $\gamma$-glutamyl transpeptidase knockout mutants $(\Delta S s$ - $g g t 1)$.

${ }^{\mathrm{z}}$ Mean values were not separable by ANOVA with an overall $P<0.05$.

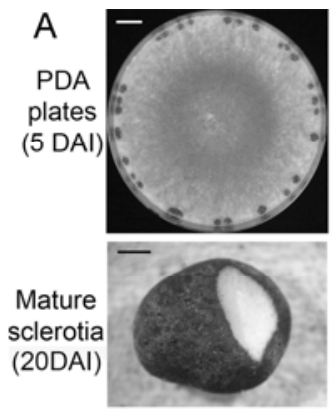

WT

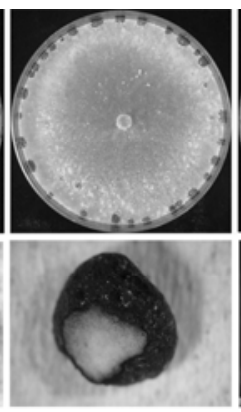

Ec

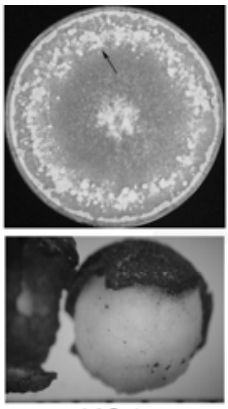

KO1

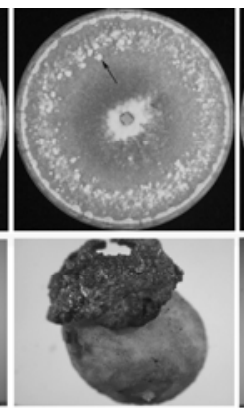

$\mathrm{KO} 2$

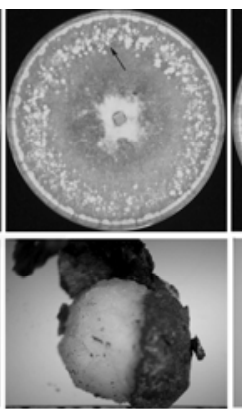

KO3

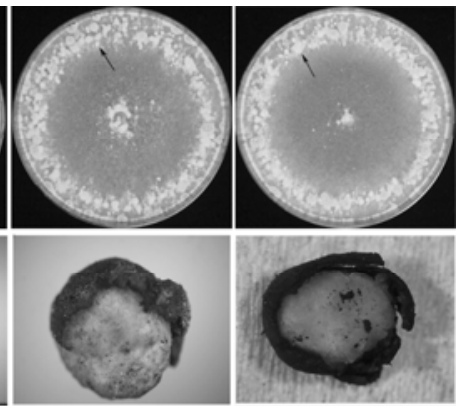

$\mathrm{KO} 4$

KO5
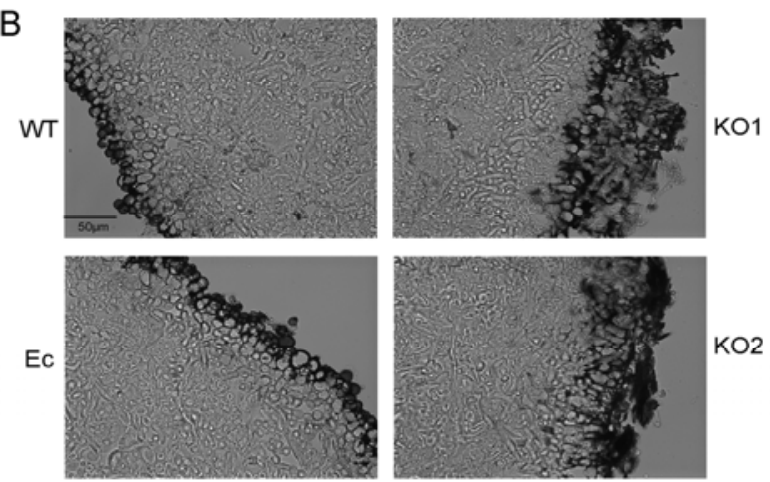

Fig. 4. Macro- and microscopic features of Sclerotinia sclerotiorum $\gamma$-glutamyl transpeptidase $(\Delta S s$-ggt1) sclerotia. A, Upper panel: potato dextrose agar (PDA) plates of the wild type (WT), an ectopic strain (Ec), and $\Delta S s$-ggtl knockout mutants (KO1 to KO5) 5 days after inoculation (DAI). Immature sclerotia of $\Delta S s$-ggt1 mutants are indicated by black arrows. Lower panel: normal mature sclerotia (stage 6) harvested from WT, Ec, and $\Delta S s$-ggt1 mutants (KO1KO5) 20 DAI. Note the easily peeled rinds of KO strains. Scale bar in upper panel = $1 \mathrm{~cm}$; scale bar in lower panel = $1 \mathrm{~mm}$. B, Micrographs from thin sections of mature (stage 6) sclerotial from WT, Ec, and two representative $\Delta$ Ss-ggt1 KO mutants (KO1 and -2). 


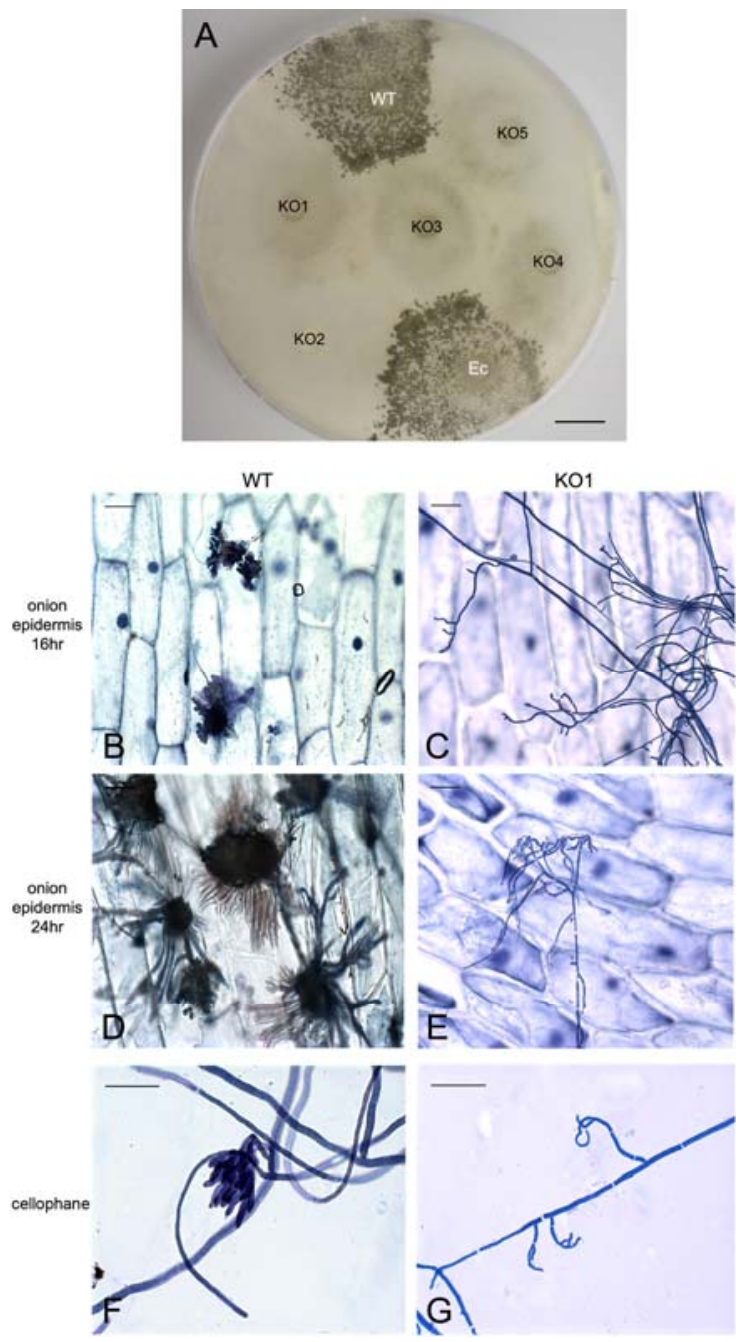

Fig. 5. Compound appressorium development by wild type (WT), ectopic strain (Ec), and Sclerotinia sclerotiorum $\gamma$-glutamyl transpeptidase knockout ( $\Delta S s$-ggtl) strains (KO1 to KO5). A, Macroscopic assessment of appressorium development on cellophane-overlain potato dextrose agar (PDA) media. Scale bar $=1 \mathrm{~cm}$. Vegetative hyphae placed on $\mathbf{B}$ to $\mathbf{E}$, onion epidermal strips and $\mathbf{F}$ to $\mathbf{G}$, cellophane-overlain PDA media. Compound appressorium and infection hyphae can be distinguished easily in the WT while appressorium initials but no distinct compound appressoria were formed by KO strains. Tissues in B were stained with 0.05\% trypan blue. Scale bars represent $50 \mu \mathrm{m}$. function $\Delta S s$-ggtl knockout mutants lacked Ggt activity, and hyperaccumulated GSH+GSSG and hydrogen peroxide during development. Phenotypic characterization of these knockout mutants indicates that Ggt1 activity is required for proper formation and function of both sclerotia and compound appressoria. These differing developmental structures play independent yet equally critical functions in the infection cycle of $S$. sclerotiorum: sclerotia for long-term survival and production of ascospore inoculum and appressoria for direct penetration of host tissues.

The most obvious manifestation of Ss-ggtl deletion in sclerotia was the thickened, disorganized rind and cortex layers that were easily removed from the underlying medulla tissue. As an apparent consequence of these tissue malformations, these sclerotia were unable to produce apothecia, thus decreasing the fitness and infection potential of these mutants. The lack of functional sclerotia precluded the assessment of $\mathrm{Ss}$ ggtl's role in post-germination apothecium development. Relating specifically to infection, compound appressoria were initiated but not properly formed by these mutants. Loss of compound appressorium development resulted in delayed and inefficient infection. These phenotypic consequences are consistent with a specific role for Ggt1 activity during developmental transitions and the maturation of these multicellular structures.

The phenotype of all five independent $S s$-ggtl mutants supports the hypothesis that this enzyme plays an important role in balancing redox during sclerotial and compound appressorium development. These mutants overaccumulate GSH+GSSG in sclerotial tissue. The associated hydrogen peroxide overaccumulation is consistent with the known function of Ggt in animal systems in maintaining redox homeostasis in the cellular environment (Zhang and Forman 2009). The lack of glutamyl and cysteinylglycine conjugates available for recycling is expected to result in excessive GSSG accumulation, consistent with our finding of excessive GSH+GSSG accumulation in $\Delta S s$-ggtl sclerotia. Whether this accumulation is extracellular or intracellular was not explored in our studies. Despite the prediction of a secretion signal in the Ss-Ggt protein, the Saccharomyces cerevisiae Ggt protein, ECM38, also contains a secretion signal but has been localized to vacuoles (Mehdi et al. 2001). This leaves the possibility of intracellular localization for the $S$. sclerotiorum Ggt1 and Ggt2 open for future analyses. The low level of $S s$-ggt2 expression in sclerotia coupled with the lack of Ggt activity in the $\Delta S s$-ggtlmutants suggests that total Ggt activity in WT sclerotia is provided by Ss-Ggt1.
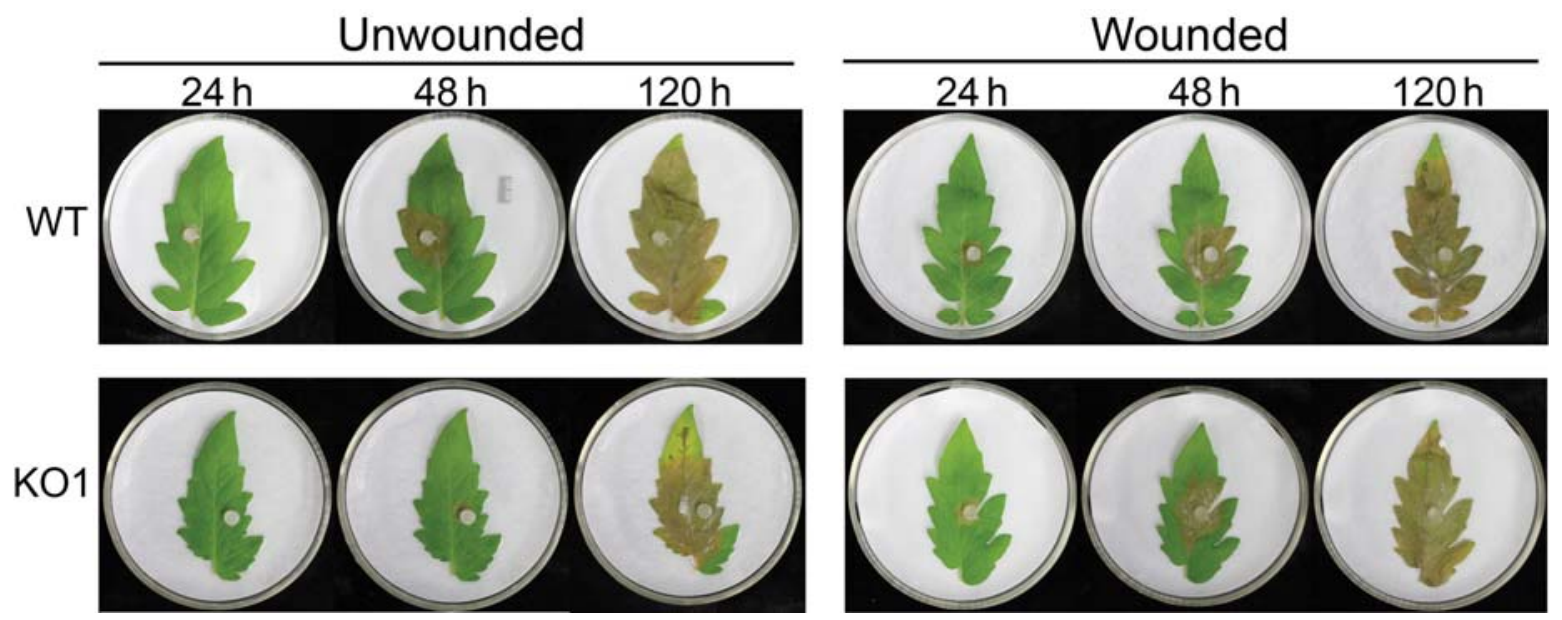

Fig. 6. Tomato leaflets infected by wild type (WT) and Sclerotinia sclerotiorum $\gamma$-glutamyl transpeptidase knockout ( $\Delta S s$-ggt1 KO1). Representative photos that best match the mean lesion area for each time point calculated for the WT and the ectopic control across three experiments and for all five $\Delta S s$-ggtl KO mutants are shown. The ruler segment is $1 \mathrm{~cm}$. 
Without sufficient GSH reductase activity to compensate for the lack of Ggt activity, an imbalance of the redox state would be expected to result in a persistent oxidative environment. This is consistent with the observation of excessive hydrogen peroxide accumulation observed in mature (stage 6) $\Delta S s$-ggt 1 sclerotia. The $S$. sclerotiorum genome is predicted to encode two GSH reductases but their activities are apparently not sufficient in this context to regenerate levels of GSH required to prevent oxidative buildup in the absence of Ss-Ggt1.

The attenuated virulence observed with the $\Delta S s$-ggtl mutants appears to be exclusively related to the associated defect in compound appressorium development. Plants that were wounded prior to infection exhibited no significant difference in infection efficiency or rate of lesion expansion when inoculated with WT or $\Delta S s$-ggtl mutants. Furthermore, no differences in host colonization or symptom development were detected among the five independent $\Delta S s$-ggtl mutants relative to the WT or ectopic control strain. These observations suggest that Ggt1 activity is dispensable during the colonization phase of $S$. sclerotiorum pathogenesis. If there is a role for Ggt activity during host colonization, it may be provided by Ss-Ggt 2 or -3 . Transcripts of $S s-g g t 2$ accumulated to a low level in infected tomato leaves and, as such, may have a function during $S$. sclerotiorum host colonization. A role for Ggt activity during host colonization could be assessed by functional characterization of the Ss-ggt 2 and -3 genes.

The capacity to regulate the external redox environment has been shown to play important roles in host infection by plantpathogenic fungi closely related to $S$. sclerotiorum. Hyphae of Monilinia fructicola treated with host phenols such as caffeic acid show increases in GSH+GSSG levels and corresponding decreases in electrochemical redox potential (Lee and Bostock 2007). When applied to conidia, both caffeic acid and reduced GSH inhibit appressorium formation without significantly affecting conidial germination. This inhibition suggests that appropriate redox regulation is required for appressorium development in M. fructicola (Lee and Bostock 2007) and that GSH may play a role in modulating this regulation. In Botrytis cinerea, another closely related necrotrophic pathogen, NADPH oxidases (Nox) encoded by bcnoxA and bcnoxB are differentially important in the infection and colonization process. bcnoxB is required for appressorium development and function whereas bcnoxA is required for post-penetration secondary lesion expansion (Segmüller et al. 2008). bcnoxA and bcnoxB are both required for sclerotium development. Nox enzymes are important endogenous generators of ROS in other fungi as well (Takemoto et al. 2007). In Magnaporthe grisea, the accumulation of ROS is pronounced during conidiospore germination and appressorium development. Deletion of NADPH oxidases noxl and nox 2 in $M$. grisea demonstrate that neither is required for appressorium development but both are required for appressorium penetration and in planta colonization, even when wound inoculated (Egan et al. 2007). Ss-Ggt1 in S. sclerotiorum appears to function as a modulator of redox homeostasis, potentially acting in concert with NADPH oxidases. Similar to bcnoxB, Ss-Ggt1 activity is restricted to specific developmental transitions. Ss-Ggt1 could potentially function downstream of or cooperatively with the $S$. sclerotiorum equivalent of $\mathrm{BcNoxB}$ as a means of balancing or eliminating the hyperoxidant environment during the normal course of development to ensure the transient nature of the hyperoxidant state. This is consistent with both gene mutations affecting sclerotial and compound appressorium development. These findings suggest that Ss-Ggt1 is not a general life-cycle redox regulator but has activity associated with developmental processes.

The data from this study support the hyperoxidant hypothesis of microbial cellular differentiation (Aguirre et al. 2005;
Hansberg and Aguirre 1990) and suggest that Ggt is one of the factors necessary to modulate the rapid accumulation of ROS and ensure the transient nature of the hyperoxidant state associated with development. The requirement for Ss-Ggt1 in both sclerotial and compound appressorium development may have a basis in the common requirement for cellular adhesion and melanin accumulation in both structures. In Neurospora crassa, a peak in extracellular accumulation of GSSG is associated with the redox imbalance following exposure of hyphae to an air interface (Toledo et al. 1991). This hyperoxidant state is concurrent and seemingly causal to hyphae-hyphae adhesion (Toledo et al. 1991). In both sclerotia and compound appressorium development, hyphae self-adhere to form their multicellular forms. In the $\Delta S s$-ggtl mutants, the increased occurrence of sclerotial initials may be the consequence of an increase in cell adhesion when sclerotial development initiates. As development proceeds, a disruption in cell-cell adhesion is localized to the outer layers of the mature sclerotia. This can be observed in the microscopic sections of sclerotia and by the ease of rind detachment when manipulated by hand. One interpretation of these observations is that the inability to decrease GSSG levels results in a hyperadhesion. This may be followed by an inability to coordinate spatial development of the outermost air-exposed layers due to a failure to establish a final reducing environment required for maturation. Similarly, the developmental defect observed in compound appressoria occurs after initiation. The characteristic hooking and bifurcation of the hypha tip occurs but is followed by further uncontrolled branching and a lack of melanin accumulation, suggesting that the final coordination of developmental maturation may also require a return to a more reducing environment.

In conclusion, these findings implicate Ss-Ggt1 and its substrates GSH and GSSG, as functioning in sclerotial and compound appressorium development. GSH, GSSG, or GSH/GSSG ratios may function as signals directly influencing signal pathways mediating sclerotium and compound appressorium development. Additionally, the redox state affected by GSH/GSSG imbalance may have direct effects on protein and non-protein thiols promoting cell adhesion and other biochemical changes required for sclerotium and compound appressorium formation. Potent inhibitors of Ggt activity have been developed recently (Han et al. 2007) and may have applications in disease control based on our findings that Ggt is required for two essential stages of the disease cycle. Further characterization of the generators and modulators of the redox systems may provide additional targets for interference in the life cycle and infectious development of this and related phytopathogenic fungi.

\section{MATERIALS AND METHODS}

\section{Culture growth.}

S. sclerotiorum WT strain 1980 was cultured on PDA plates. PDA cultures of $\Delta S s$-ggt 1 mutants $(\mathrm{KO} 1=\mathrm{Ss}$-ggt1-3.10, KO2 = Ss-ggt1-2.3, KO3 = Ss-ggt1-2.16, KO4 = Ss-ggt1-3.12, and $\mathrm{KO} 5=$ Ss-ggt1-3.26) and ectopic strain 3.29 (ectopic) were amended with hygromycin (final concentration $=100 \mu \mathrm{g} / \mathrm{ml}$ ). Sclerotium development stages (S1-S6) previously described by $\mathrm{Li}$ and Rollins (2009) were used to define the developmental stages of sclerotia in this study. These stages are $\mathrm{S} 1=$ initiation, $\mathrm{S} 2=$ condensation, $\mathrm{S} 3=$ enlargement, $\mathrm{S} 4=$ consolidation, $\mathrm{S} 5=$ pigmentation, and $\mathrm{S} 6=$ maturation.

\section{Generation of Ggt gene deletion mutants ( $\Delta S$ Ss-ggt1).}

A split-marker gene replacement method (Fairhead et al. 1996; Fu et al. 2006) was used to obtain multiple, independent Ss-ggtl (SS1G_14127.3) gene-replacement knockout mutants. 
The strategy for gene replacement is shown in Figure 2. A plasmid containing a hygromycin resistance cassette flanked with approximately $1.2 \mathrm{~kb}$ of $5^{\prime}$ - and $3^{\prime}$-UTR sequence from the WT Ss-ggtl locus (ggt-5'+hph+3') was constructed based on the method described by $\mathrm{Li}$ and Rollins (2010). Primer pairs 5'ggtMu-L1 (5'-TTCAAAAGGGCTGAGTGTGA-3') and 5'ggtMu-R1 (5'-AGGCGCGCCCAACCCGGGAGAATGAGT TA-3'), and $3^{\prime}$ ggtMu-L1 (5'-AGGCGCGCCGGGGTTTTAAT CTAGGATACGG-3') and 3'ggtMu-R1 (5'-GAAAGGTGGTG GACTTTGGA-3') were used for polymerase chain reaction (PCR) to acquire 5'- and 3'-UTR amplicons, respectively, of the Ss-ggtl locus from WT S. sclerotiorum genomic DNA. AscI restriction endonuclease recognition sites (underlined) were incorporated into the primers to facilitate cloning. The detailed steps for construction of the ggt $-5^{\prime}+h p h+3^{\prime}$ construct followed the previously described procedure ( $\mathrm{Li}$ and Rollins 2010). Two "split" fragments (5'-UTR joined with the 5' hph sequence " $h y$ " and the second overlapping $h p h$ " $y g$ " fragment joined with the $3^{\prime}$-UTR) (Fig. 2) were transformed into WT protoplasts. These fragments were derived from amplification of ggt- $5^{\prime}+\mathrm{hph}+3^{\prime}$ using primer pairs ggtL (5'-TTCAAAAGGG CTGAGTGTGA-3') and hy (5'-AAATTGCCGTCAACCAAG CTC-3') and yg (5'-TTTCAGCTTCGATGTAGGAGG-3') and ggtR (5'-GAAAGGTGGTGGACTTTGGA-3'). Transformation of WT protoplasts was done according to the method described by Rollins (2003). Constructed plasmids were all transformed into Escherichia coli DH5 $\alpha$ for propagation. Plasmid isolation, enzyme digestion, gel electrophoresis, DNA fragment purification, and ligation were conducted using standard procedures (Sambrook and Russell 2001) or according to the manufacturer's instruction.

\section{Nucleic acid isolation and hybridization.}

Genomic DNA and RNA were isolated, DNA digested, and gel electrophoresis performed according to standard procedures (Sambrook and Russell 2001). Southern hybridizations were conducted according to the descriptions of Rollins (2003). Restriction endonuclease sites and probes for Southern hybridization are shown in Figure 2. Nonradioactive Northern hybridizations were carried out according to the procedures reported by Solanas and associates (2001). Primer pairs (number 137) ggtCDSprob-L (TGCAAAGAATGGAGCTGTTG) and (number 138) ggtCDSprob-R (5'-ATGGTCCCATTTGCTTT CTG-3') for ggt1, (number 781) ggt2CDSprobe-L (5'-TAG GCG GAG GTG GTT TTA TG-3') and (number 782) ggt2CDSprobe-R (5'-ACG AGC ACC AGG ACG AAC AT-3') for $g g t 2$, and (number 783) ggt3CDSprobe-L(5'-ACA CCG GAA AAA TCG CTG AA-3') and (number 784)ggt3CDSprobe-R(5'-CCT TTT ACC AGG AGC GAT GA-3') for ggt3 were used to synthesize Northern hybridization probes by PCR. Each PCR reaction mixture contained $10 \times$ digoxigenin (DIG) labeling mixture from DIG High Prime DNA labeling and detection starter kit I Roche, Indianapolis, IN, U.S.A.), $2.5 \mathrm{mM}$ $\mathrm{MgCl}_{2}, 0.2 \mathrm{mM}$ dNTPs, $1.6 \mu \mathrm{M}$ forward primers, $1.6 \mu \mathrm{M}$ reverse primers, $10 \times$ Taq buffer, $0.65 \mathrm{U}$ of Taq polymerase (New England Biolabs, Inc., Ipswich, MA, U.S.A.), 100 ng of DNA template, and $\mathrm{H}_{2} \mathrm{O}$ made up to the final volume of $25 \mu \mathrm{l}$. The PCR program was $95^{\circ} \mathrm{C}$ for $4 \mathrm{~min} ; 35$ cycles at $95^{\circ} \mathrm{C}$ for $30 \mathrm{~s}$, $60^{\circ} \mathrm{C}$ for $30 \mathrm{~s}$, and $72^{\circ} \mathrm{C}$ for $1.5 \mathrm{~min}$; followed by $72^{\circ} \mathrm{C}$ for 10 min. Labeling efficiency was determined as described in the instruction manual (Roche). Final concentration of probe in hybridization buffer was $25 \mathrm{ng} / \mathrm{ml}$.

\section{GSH+GSSG accumulation assay.}

Stage 4 to 5 sclerotia and stage 6 sclerotia from WT, ectopic, and Ss-ggtl knockout strains were harvested from PDA plates with forceps. Lyophilized sclerotia $(100 \mathrm{mg})$ from each strain were weighed and homogenized for GSH+GSSG extraction. GSH extraction and determination were conducted using the ApoGSHTM glutathione colorimetric detection kit (Biovision Research Products, Mountain View, CA, U.S.A.) following the manufacturer's instruction.

\section{Enzyme extraction and Ggt activity assay.}

To obtain protein extracts for Ggt activity measurement, lyophilized sclerotia from WT, ectopic, and knockout strains were homogenized in protein extraction buffer as previously described (Li and Rollins 2009). Ggt activity was measured spectrophotometrically based on a modified protocol of Ikeda (2000). Ggt reaction mixture $(100 \mu \mathrm{l})$ was added to wells of a 96-well microtiter plate containing $100 \mathrm{mM}$ Tris- $\mathrm{HCl}(\mathrm{pH}$ 8.0), $1 \mathrm{mM} \gamma$-GPNA, $20 \mathrm{mM}$ glycylglycine, and $10 \mu \mathrm{l}$ of total protein extract. The plate was incubated at $37^{\circ} \mathrm{C}$ during plate reading. Absorbance values were recorded every $30 \mathrm{~s}$ for 10 min at $410 \mathrm{~nm}$ using a Bio-Rad microplate reader Model 680 with Microplate Manager Version 5.2 Software (Bio-Rad, Hercules, CA, U.S.A.). Ggt activity was calculated using the equation described by Huseby and Strömme (1974).

\section{Hydrogen peroxide detection.}

To detect hydrogen peroxide accumulation in sclerotia, sclerotia were weighed, sectioned (approximately $30 \mu \mathrm{m}$ ), and immersed into freshly prepared DAB (D5637; Sigma-Aldrich, St. Louis) solution at $1.25 \mathrm{mg} \mathrm{ml}^{-1}$. DAB was dissolved in $\mathrm{H}_{2} \mathrm{O}$ and adjusted to $\mathrm{pH} 3.8$ with $\mathrm{HCl}$ prior to use. Submerged sclerotia slices were infiltrated under vacuum for $5 \mathrm{~min}$ and then shaken at $50 \mathrm{rpm}$ for $4 \mathrm{~h}$. Photographs were taken for a qualitative assessment of DAB staining. To quantitatively determine $\mathrm{H}_{2} \mathrm{O}_{2}$ concentrations, DAB solution absorbance was measured at $450 \mathrm{~nm}$. Concentration values were extrapolated from a standard curve produced from known concentrations of $\mathrm{H}_{2} \mathrm{O}_{2}$ incubated for the same time period in DAB solution.

\section{Compound appressorium and plant infection assays.}

The production of compound appressoria was assayed in vitro using a cellophane-media overlay technique. A single sheet ( $9 \mathrm{~cm}$ diameter) of autoclaved cellophane (Bio-Rad) was overlain on PDA. Sterile deionized water $(500 \mu \mathrm{l})$ was applied to the cellophane surface and spread with a glass rod until a smooth surface was achieved and all the water was absorbed. Hyphae-colonized agar plugs $(0.5 \mathrm{~cm}$ diameter $)$ were taken from the expanding margin of PDA cultures and placed hyphae-side down on the cellophane in evenly spaced rows. Plates were incubated on the lab bench (approximately $22^{\circ} \mathrm{C}$ ) under ambient fluorescent light conditions (approximately 2.8 $\mu \mathrm{mol} \mathrm{m} \mathrm{m}^{-2} \mathrm{~s}^{-1}, 12 \mathrm{~h}$ per day). Following a 48-h incubation period, the bottoms of the plates were photographed.

Inoculation of unwounded tomato leaflets was conducted as previously described (Rollins 2003). For the wounded treatment, a $0.5-\mathrm{cm}$ cut was made through the abaxial and adaxial surface of each leaflet with a scalpel blade and the inoculum plug placed directly on top of the cut. Each leaflet was digitally photographed at 24 and $48 \mathrm{~h}$ postinoculation. The images were imported to the Spot Advanced Software program (Diagnostic Instruments, Sterling Heights, MI, U.S.A.) and lesion areas calculated by tracing the necrotic area, calculating the area, and subtracting the inoculum plug area. The lesion area for each fungal strain in wounded and unwounded treatments was calculated for 24 and $48 \mathrm{~h}$ postinoculation time points. An analysis of variance was performed with the mean lesion areas from each treatment time point; a $P$ value $<0.05$ was considered significant. Differences in lesion area among strains within a given treatment time point was determined by the Bonferroni (Dunn) $t$ test $(\alpha=0.05)$. Each experiment included 
three leaflets inoculated with each fungal strain and the experiment was repeated three times. Results of a representative replicate from one experiment most closely matching the mean lesion area of the WT and the Ss-ggtl KO mutants across all experiments are shown in Figure 6.

\section{Apothecia production.}

To test for carpogenic germination, mature sclerotia of $\Delta S s$ ggtl mutants and WT were produced, surface sterilized, and incubated as previously described ( $\mathrm{Li}$ and Rollins 2010).

\section{ACKNOWLEDGMENTS}

M. Li was supported by a Graduate Alumni Fellowship provided by the University of Florida. We thank P. Harmon and A. Wright for assistance with statistical analysis and U. Benny for laboratory support.

\section{LITERATURE CITED}

Abawi, G. S., Polach, F. J., and Molin, W. T. 1975. Infection of bean by ascospores of Whetzelinia sclerotiorum. Phytopathology 65:673-678.

Aguirre, J. Riosmomberg, M., Hewitt, D., and Hansberg, W. 2005. Reactive oxygen species and development in microbial eukaryotes. Trends Microbiol. 13:111-118.

Akutsu, K., Ko, K., and Misato, T. 1983. Nuclear behavior from conidial germination to formation of the secondary appressoria of Botrytis cinerea. Ann. Phytopathol. Soc. Jpn. 49:361-366.

Amselem, J., Cuomo, C. A., van Kan, J. A. L., Viaud, M., Benito, E. P., Couloux, A., Coutinho, P. M., de Vries, R. P., Dyer, P. S., Fillinger, S., Fournier, E., Gout, L., Hahn, M., Kohn, L., Lapalu, N., Plummer, K. M., Pradier, J.-M., Quévillon, E., Sharon, A., Simon, A., ten Have, A., Tudzynski, B., Tudzynski, P., Wincker, P., Andrew, M., Anthouard, V., Beever, R. E., Beffa, R., Benoit, I., Bouzid, O., Brault, B., Chen, Z., Choquer, M., Collémare, J, Cotton, P., Danchin, E. G., Da Silva, C., Gautier, A., Giraud, C., Giraud, T., Gonzalez, C., Grossetete, S., Güldener, U., Henrissat, B., Howlett, B. J., Kodira, C., Kretschmer, M., Lappartient, A., Leroch, M., Levis, C., Mauceli, E., Neuvéglise, C., Oeser, B., Pearson, M., Poulain, J., Poussereau, N., Quesneville, H., Rascle, C., Schumacher, J., Ségurens, B., Sexton, A., Silva, E., Sirven, C., Soanes, D. M., Talbot, N. J., Templeton, M., Yandava, C., Yarden, O., Zeng, Q., Rollins, J. A., Lebrun, M.-H., and Dickman, M. 2011 Genomic analysis of the necrotrophic fungal pathogens Sclerotinia sclerotiorum and Botrytis cinerea. PLoS Genet. 7:e1002230.

Backhouse, D., and Willetts, H. J. 1987. Development and structure of infection cushions of Botrytis cinerea. Trans. Br. Mycol. Soc. 89:8995.

Blokhina, O., Virolainen, E., and Fagersted, K. V. 2003. Antioxidants, oxidative damage and oxygen deprivation stress: A review. Ann. Bot. 91:179-194.

Boland, G. J., and Hall, R. 1994. Index of plant hosts of Sclerotinia sclerotiorum. Can. J. Plant Pathol. 16:93-108.

Bolton, M. D., Thomma, B. P. H. J., and Nelson, B. D. 2006. Sclerotinia sclerotiorum (Lib.) de Bary: Biology and molecular traits of a cosmopolitan pathogen. Mol. Plant Pathol. 7:1-16.

Chet, I., and Henis, Y. 1975. Sclerotial morphogenesis in fungi. Annu. Rev. Phytopathol. 13:169-192.

Cruickshank, R. H., and Wadea, G. C. 1992. Production of appressoria by Monilinia fructicola Mycol. Res. 96:425-428.

Egan, M. J., Wang, Z., Jones, M. A., Smirnoff, N., and Talbot, N. J. 2007. Generation of reactive oxygen species by fungal NADPH oxidases is required for rice blast disease. Proc. Natl. Acad. Sci. U.S.A. 104:1177211777.

Emmett, R. W., and Parbery, D. G. 1975. Appressoria. Annu. Rev. Phytopathol. 13:147-167.

Erental, A., Harel, A., and Yarden, O. 2007. Type 2A phosphoprotein phosphatase is required for asexual development and pathogenesis of Sclerotinia sclerotiorum. Mol. Plant-Microbe Interact. 20:944-954.

Fairhead, C., Liorente, B., Denis, F., Soler, M., and Dujon, B. 1996. New vectors for combinatorial deletions in yeast chromosomes and for gap-repair cloning using 'split-marker' recombination. Yeast 12:14391458.

Franco, R., and Cidlowski, J. A. 2009. Apoptosis and glutathione: Beyond an antioxidant. Cell Death Differ. 16:1303-1314.

Fu, J., Hettler, E., and Wickes, B. L. 2006. Split marker transformation increases homologous integration frequency in Cryptococcus neoformans. Fungal Genet. Biol. 43:200-212.

Georgiou, C. D. 1997. Lipid peroxidation in Sclerotium rolfsii: A new look into the mechanism of sclerotial biogenesis in fungi. Mycol. Res. 101:460-464

Georgiou, C. D., Patsoukis, N., Papapostolou, L., and Zervoudakis, G. 2006. Sclerotial metamorphosis in filamentous fungi is induced by oxidative stress. Integr. Comp. Biol. 46:691-712.

Han, L., Hiratake, J., Kamiyama, A., and Sakata, K. 2007. Design, synthesis, and evaluation of gamma-phosphono diester analogues of glutamate as highly potent inhibitors and active site probes of gamma-glutamyl transpeptidase. Biochemistry 46:1432-1447.

Hansberg, W., and Aguirre, J. 1990. Hyperoxidant states cause microbial cell differentiation by cell isolation from dioxygen. J. Theor. Biol. 142:201-221

Huseby, N. E., and Stromme, J. H. 1974. Practical points regarding routine determination of gamma-glutamyl transferase (gamma-GT) in serum with a kinetic method at 37 degrees C. Scand. J. Clin. Lab Invest. 34:357-363.

Ikeda, Y. 2000. $\gamma$-Glutamyl transpeptidase. Pages 86-87 in: Experimental Protocols for Reactive Oxygen and Nitrogen Species. N. Taniguchi and J. M. C. Gutteridge, eds. Oxford University Press, U.S.A., New York.

Jurick, W. M., and Rollins, J. A. 2007. Deletion of the adenylate cyclase ( $(s a c 1)$ gene affects multiple developmental pathways and pathogenicity in Sclerotinia sclerotiorum. Fungal Genet. Biol. 44:521-530.

Krance, S. M., Keng, P. C., Palis, J., and Ballatori, N. 2010. Transient glutathione depletion determines terminal differentiation in HL-60 cells Oxid. Med. Cell Longev. 3:53-60.

Lee, M.-H., and Bostock, R. M. 2007. Fruit exocarp phenols in relation to quiescence and development of Monilinia fructicola infections in Prunus: A role for cellular redox? Phytopathology 97:269-277.

Li, M., and Rollins, J. A. 2009. The development-specific protein (Ssp1) from Sclerotinia sclerotiorum is encoded by a novel gene expressed exclusively in sclerotium tissues. Mycologia 101:34-43.

Li, M., and Rollins, J. A. 2010. The development-specific ssp1 and ssp2 genes of Sclerotinia sclerotiorum encode lectins with distinct yet compensatory regulation. Fungal Genet. Biol. 47:531-538.

Lum, G., and Min, X. J. 2011. FunSecKB: The fungal secretome knowledgebase database J. Biol. Database Curat. doi:10.1093/database/bar001. Published online.

Lumsden, R. D., and Dow, R. L. 1973. Histopathology of Sclerotinia sclerotiorum infection of bean. Phytopathology 63:708-715.

May, M. J., Vernoux, T., Leaver, C., Van Montagu, M., and Inzé, D. 1998. Glutathione homeostasis in plants: Implications for environmental sensing and plant development. J. Exp. Bot. 49:649-667.

Mehdi, K., Thierie, J., and Penninckx, M. 2001. $\gamma$-Glutamyl transpeptidase in the yeast Saccharomyces cerevisiae and its role in the vacuolar transport and metabolism of glutathione. Biochem. J. 359:631-637.

Meister, A., and Anderson, M. E. 1983. Glutathione. Annu. Rev. Biochem. 52:711-760.

Pallardó, F. V., Markovic, J., García, J. L., and Viňa, J. 2009. Role of nuclear glutathione as a key regulator of cell proliferation. Mol. Aspects Med. 30:77-85.

Papapostolou, I., and Georgiou, C. D. 2010. Hydrogen peroxide is involved in the sclerotial differentiation of filamentous phytopathogenic fungi. J. Appl. Microbiol. 109:1929-1936.

Patsoukis, N., and Georgiou, C. D. 2007. Effect of glutathione biosynthesis-related modulators on the thiol redox state enzymes and on sclerotial differentiation of filamentous phytopathogenic fungi. Mycopathologia 163:335-347

Patsoukis, N., and Georgiou, C. D. 2008. Thiol redox state and related enzymes in sclerotium-forming filamentous phytopathogenic fungi. Mycol. Res. 112:602-610.

Purdy, L. H. 1958. Some factors affecting penetration and infection by Sclerotinia sclerotiorum. Phytopathology 48:605-609.

Purdy, L. H. 1979. Sclerotinia sclerotiorum: History, diseases and symptomatology, host range, geographic distribution, and impact. Phytopathology 69:875-880.

Rollins, J. A. 2003. The Sclerotinia sclerotiorum pacl gene is required for sclerotial development and virulence. Mol. Plant-Microbe Interact. 16:785-795.

Sambrook, J., and Russell, D. W. 2001. Molecular Cloning: A Laboratory Manual. Cold Spring Harbor Laboratory Press, Cold Spring Harbor, NY, U.S.A.

Scott, B., and Eaton, C. J. 2008. Role of reactive oxygen species in fungal cellular differentiations. Curr. Opin. Microbiol. 11:488-493.

Segmüller, N., Kokkelink, L., Giesbert, S., Odinius, D., van Kan, J., and Tudzynski, P. 2008. NADPH Oxidases are involved in differentiation and pathogenicity in Botrytis cinerea. Mol. Plant-Microbe Interact. 21:808-819.

Solanas, M., Moral, R., and Escrich, E. 2001. Improved non-radioactive Northern blot protocol for detecting low abundance mRNAs from mam- 
malian tissues. Biotechnol. Lett. 23:263-266.

Takemoto, D., Tanaka, A., and Scott, B. 2007. NADPH oxidases in fungi: Diverse roles of reactive oxygen species in fungal cellular differentiation. Fungal Genet. Biol. 44:1065-1076.

Tariq, V. N., and Jeffries, P. 1984. Appressorium formation by Sclerotinia sclerotiorum: Scanning electron microscopy. Trans. Br. Mycol. Soc. 82:645-651.

Tate, S. S., and Meister, A. 1981. $\gamma$-Glutamyl transpeptidase-catalytic, structural and functional aspects. Mol. Cell. Biochem. 39:357-368.

Toledo, I., Noronha-Dutra, A. A., and Hansberg, W. 1991. Loss of $\mathrm{NAD}(\mathrm{P})$-reducing power and glutathione disulfide excretion at the start of induction of aerial growth in Neurospora crassa. J. Bacteriol. 173:3243-3249.

Zhang, H., and Forman, H. J. 2009. Redox regulation of gamma-glutamyl transpeptidase. Am. J. Respir. Cell Mol. Biol. 41:509-515.

\section{AUTHOR-RECOMMENDED INTERNET RESOURCE}

Broad Institute $S$. sclerotiorum genome sequence database: www.broad.mit.edu/annotation/genome/sclerotinia_sclerotiorum/ FeatureSearch.html 